\title{
Globe
}

Revue internationale d'études québécoises

\section{Mary Jean Green : Women \& Narrative Identity. Rewriting the Quebec National Text. Montréal, McGill-Queen's University Press, 2001}

\section{Lucie Guillemette et Cynthia Lévesque}

Volume 6, numéro 2, 2003

URI : https://id.erudit.org/iderudit/1000825ar

DOI : https://doi.org/10.7202/1000825ar

Aller au sommaire du numéro

Éditeur(s)

Globe, Revue internationale d'études québécoises

ISSN

1481-5869 (imprimé)

1923-8231 (numérique)

Découvrir la revue

Citer ce compte rendu

Guillemette, L. \& Lévesque, C. (2003). Compte rendu de [Mary Jean Green : Women \& Narrative Identity. Rewriting the Quebec National Text. Montréal, McGill-Queen's University Press, 2001]. Globe, 6(2), 186-192.

https://doi.org/10.7202/1000825ar d'utilisation que vous pouvez consulter en ligne.

https://apropos.erudit.org/fr/usagers/politique-dutilisation/ 
date, insérées organiquement dans les réseaux internationaux, et qui travaillent dans des champs dont, hors l'Université, aucune institution ne peut se charger? À l'heure où le monde s'est ému devant les événements tragiques que sont le saccage des grands bouddhas d'Afghanistan et le pillage des musées d'Irak, conservatoires de l'héritage mésopotamien, qu'il me soit permis d'exprimer le souhait que nous sachions mieux sensibiliser le grand public à ces entreprises fondamentales que mènent les équipes de ces secteurs ; peut-être alors nos universités s'apercevrontelles qu'elles ne doivent plus menacer ces trésors.

Dans une postface qui se lit comme un poème, Michel Despland se demande : "L'étude de la religion au Québec, comment? Pourquoi ? Comme on entreprend un voyage, répond-il, et pour les mêmes raisons, rencontrer l'autre et l'ailleurs, rester soi-même tout en s'ouvrant à l'universel. Chers collègues, merci pour ce beau livre.

Lucia Ferretti

Centre interuniversitaire d'études québécoises

Université du Québec à Trois-Rivières

\section{Mary Jean Green}

Women \& Narrative Identity.

Rewriting the Quebec National Text.

Montréal, McGill-Queen's University Press, 2001.

Dans l'ouvrage Women \& Narrative Identity, Mary Jean Green retrace le parcours de l'écriture des femmes au Québec, depuis le XIX ${ }^{e}$ siècle jusqu'à aujourd'hui. Plus précisément, il s'agit pour l'auteure de procéder à " une relecture féministe de la tradition littéraire au Québec ". Employé dans le sens où l'entend Adrienne Rich lorsqu'elle parle de revision, le terme " relecture "constitue une notion clé au plan méthodologique dans la mesure où Green entreprend de "revisiter" le roman identitaire des femmes afin de cerner leur contribution au développement de la culture québécoise. Dans l'introduction, l'auteure avance l'hypothèse que le genre romanesque au Québec a joué un rôle capital dans la construction d'une identité nationale et que les femmes ont élaboré, dans un contexte idéologiquement marqué par la survivance de la race canadiennefrançaise, depuis la fin du XIX $x^{e}$ siècle jusqu'à la Révolution tranquille, des 
fictions où elles ont pu non seulement se faire entendre, mais aussi développer des univers narratifs qui à la fois rendent compte de leur dépossession en tant que sujets féminins et subvertissent les paradigmes imposés par le discours masculin.

L'ouvrage est composé de cinq chapitres dont la longueur varie entre 19 et 31 pages. Chacune des parties est consacrée à une époque déterminante de l'histoire littéraire du Québec, revue à la faveur d'une lecture féministe des écrits des femmes. Au fil de sa démonstration, Green pose les articulations de son cadre théorique d'interprétation qui s'inspire des travaux de féministes issues de la tradition anglo-saxonne dont Nancy Miller, Elaine Showalter, Sandra M. Gilbert, Susan Gubar et Adrienne Rich. À partir de son analyse des textes majeurs signés par des femmes, Green montre comment ces dernières ont su s'imposer en s'écartant des conventions et des normes hégémoniques prescrites par la société des hommes. Ces écrivaines, d'abord marginalisées par l'institution littéraire canadienne-française, sont parvenues progressivement à exprimer leur voix et à redéfinir leur identité en réécrivant l'histoire des femmes des générations précédentes, des femmes qu'elles percevaient comme soumises et passives.

Le premier chapitre, qui s'intitule "Writing Passion Into the National Text ", concerne la naissance de la littérature féminine québécoise à la fin du $\mathrm{xIX}^{e}$ siècle. L'auteure y trace un portrait sociologique de la littérature à travers lequel s'incarne la figure pour le moins autoritaire de l'abbé Henri-Raymond Casgrain. Imposant de sévères contraintes aussi bien de type formel que thématique aux écrivains, l'homme de lettres désirait coûte que coûte assurer l'établissement d'une littérature nationale canadienne-française où primerait l'idéologie cléricale. Green rappelle par le fait même l'intransigeance de la société de l'époque à l'endroit des écrivaines. Confinée à la sphère du privé, la femme est réduite au silence; l'acte d'écriture ou tout autre geste s'écartant des tâches domestiques est perçu comme une entrave au bon fonctionnement du foyer, voire une négligence impardonnable. Au fil du premier chapitre, l'étude de textes de Laure Conan, dont Angéline de Montbrun, souligne l'émergence d'une voix féminine exprimant ses passions intimes. Ce faisant, Green évoque les singularités de l'écriture romanesque de Conan, qui s'est imposée dans les lettres québécoises en dérogeant aux paramètres circonscrits essentiellement par l'abbé Casgrain. L'utilisation de la forme épistolaire et du journal intime, qui permettent 
d'emblée l'inscription d'une subjectivité féminine qui se raconte et qui se dit, constituait à l'époque une infraction aux prescriptions génériques, la voix collective et impersonnelle ayant préséance sur la voix individuelle. L'auteure rappelle également l'implication sociale et politique ainsi que le désir d'indépendance des personnages féminins mis en scène dans les textes de Laure Conan, des femmes incapables de se soumettre au rôle social qu'on leur assignait : "Through a profound understanding of the dominant nationalist ideology of ber particular place and time and an apparently sincere allegiance to its goals, Laure Conan was able, in Angeline de Montbrun and the historical novels that followed it, to make a place for women - and women fantasies and desires - in Quebec's developing "national litterature". (p. 22). En bon censeur, Casgrain a d'ailleurs tôt fait de critiquer la présence flagrante des passions ainsi que l'attitude inacceptable, selon lui, de protagonistes qui aspirent à l'autonomie. Il n'en demeure pas moins que la légitimation des écrits de Conan s'est opérée en vertu de la valeur historique et du caractère spirituel qu'on a consenti à leur reconnaitre. Selon Green, cette première manifestation d'autonomie, qui s'incarne tant dans le comportement de Conan en tant qu'auteure que dans celui des personnages féminins qu'elle a créés, ouvre la voie aux écrivaines québécoises.

Comme l'expose le deuxième chapitre "Women and the Romance of the Land ", la première moitié du $\mathrm{xx}^{\mathrm{e}}$ siècle réduira les écrivaines au silence. Au sein de l'institution littéraire tout comme dans l'institution familiale, note Green, la femme s'efface. Le roman du terroir, qui demeure le genre prédominant, est inféodé à l'idéologie rurale et patriarcale privilégiant les thèmes de la patrie, de la terre et de la sédentarité, idéologie qui réduit la femme à un être de reproduction conçu pour assurer la survie de la race. À la suite d'un bref exposé qui reprend les faits saillants de l'histoire littéraire, la critique propose une étude des textes de deux auteures qui ont façonné l'écriture des femmes. Il s'agit de Gabrielle Roy et de Germaine Guèvremont. Green analyse la façon dont les personnages féminins campés par les romancières affrontent les réalités sociales du monde autour duquel ils gravitent. Force est de constater que la société rurale traditionnelle ne correspond plus à la réalité québécoise, marquée de plus en plus par l'urbanisation et l'industrialisation. Nul doute que pour la citoyenne évoluant dans un contexte où percent les avatars du modernisme, la soumission et l'effacement sont des traits propres à un comportement guidé par la servitude, autant d'attitudes qui empêchent l'accès aux sphères décisionnelles. Comme l'explique Green, 


\section{RECENSIONS}

Roy et Guèvremont exposent l'insatisfaction des femmes et leur aspiration à se détacher du rôle féminin dicté par les conventions patriarcales. Green décrit alors une période transitoire où les personnages mis en scène par les deux écrivaines convoitent d'autres horizons, un sentiment dont se réclament une majorité de femmes au Québec. Toujours selon l'auteure, les avancées des écrivaines au Canada français davantage orientées vers la quête identitaire se distinguent des activités des militantes pour obtenir le droit de vote durant les premières décennies du $\mathrm{xx}^{e}$ siècle. Toutefois, entre Laure Conan et Gabrielle Roy se sont manifestées quelques voix féminines au sein de l'institution littéraire, que Green évoque un peu rapidement. Rappelant la réception plutôt violente des textes d'Éva Sénécal et de Jovette Bernier, l'auteure ignore le travail de jeunes poétesses, comme Alice Lemieux et Simone Routier, qui ont exploré de nouvelles formes d'expression mettant en scène une subjectivité féminine.

Dans le troisième chapitre, consacré aux femmes et à la révolution, Green décrit le contexte politique, social et culturel à l'aube de la Révolution tranquille. Un changement radical s'amorce au cœur de toutes les sphères de la société : les Québécois ressentent le besoin d'afficher leurs différences et leur indépendance, tant sur le plan personnel que politique. Impossible, dès lors, de penser la littérature en dehors d'un contexte orienté vers des transformations sociales majeures. L'auteure rappelle que deux écrivaines québécoises, Marie-Claire Blais et Anne Hébert, sont parvenues à intégrer à leurs fictions une quête vers l'autonomie personnelle et vers la libération sexuelle dans les coulisses d'un discours social où prévalait la dynamique politique de l'indépendance et de la souveraineté du Québec. Chacune à leur manière, ces écrivaines mettent en scène une dimension spécifique de l'expérience des femmes au Québec : les conflits intergénérationnels, l'incompréhension et l'incommunicabilité, la révolte. Au terme d'une analyse détaillée de Kamouraska, Green conclut cependant que le discours des romancières, loin d'être marginal, rejoint celui tenu par la majorité au Québec : deux ordres discursifs qui réclament une libération, un affranchissement face à l'oppression d'une société programmée avant tout pour " surveiller et punir ", pour reprendre les termes de Michel Foucault : "Kamouraska illustrates the way in which the desire for liberation and self-affirmation on the part of Quebec society as a whole coincided in the 1960 s with the desire of Quebec women for liberation from the traditional patriarchal ideology. (p. 102). Cette troisième partie se distingue des précédentes 


\section{REVUE INTERNATIONALE D'ÉTUDES QUÉBÉCOISES}

dans la mesure où l'accent est mis sur le contexte politique, élément nécessaire à la compréhension de l'évolution de l'écriture des femmes, si l'on songe aux importants changements qui sont survenus durant cette période en ce qui a trait à l'émancipation du sujet féminin. L'analyse menée par Green souscrit à bien des égards aux travaux de Diane Lamoureux exposés dans L'amère patrie (2001) et Malaises identitaires (1999), essais consacrés aux rapports entre le féminisme et le nationalisme québécois. Cette partie est sans doute la mieux réussie dans la mesure où le propos examinant les rapports entre le littéraire et le politique est bien étayé.

$\mathrm{Si}$ les auteures québécoises se rallient au discours politique valorisant les thèmes nationalistes et indépendantistes avant 1980 , il en sera autrement après l'échec référendaire. À l'instar de certains écrivains qui affectionnent le roman psychologique et les textes intimistes, elles se détournent du " nous "homogénéisant qui entre en contradiction avec la subjectivité féminine qui, elle, se veut plurielle, hétérogène et ouverte au changement. Green montre, au sein du quatrième chapitre, intitulé "Rewriting the Narratives of Identity", l'aboutissement de la lutte des écrivaines pour atteindre leur autonomie dans le champ littéraire. Alors qu'elle explore les textes représentatifs de l'écriture féministe au Québec, l'auteure montre la nouvelle quête identitaire qui absorbe le sujet féminin, une démarche visant à redonner aux femmes du passé la voix qu'elles n'ont pu articuler : "For many Quebec women writers of the 1980 s, the opening up of the subject was also an opening into bistory, and the multiple voices that circulate in their texts include those emerging from a long obscured past." (p. 122). La liberté quant au mélange des genres littéraires, l'amalgame de la fiction et de la théorie, l'exploration du langage sous un angle formel, la valorisation de la généalogie féminine et la déconstruction de l'Histoire sont autant de procédés littéraires utilisés pour façonner l'identité des femmes au Québec. Green se penche également sur l'insertion du mouvement féministe dans le paysage littéraire québécois en évoquant quelques textes significatifs, dont ceux de France Théoret (Nous parlerons comme on écrit, Entre raison et déraison), d'Arlette Cousture (Les filles de Caleb) et de Madeleine Ouellette-Michalska (La maison Trestler).

Le dernier chapitre, consacré aux "nouveaux récits de l'identité ", présente la situation culturelle qui prévaut dans le Québec du Xxi ${ }^{e}$ siècle, une situation où la culture est diversifiée, compte tenu de la réalité 


\section{RECENSIONS}

multiethnique qui confère un nouveau visage à la société québécoise. Cependant, cette pluralité de voix semble poser problème quant à la quête identitaire menée par les femmes nées au Québec, considérées comme "de souche". Plusieurs textes représentatifs d'une écriture migrante déprécient en effet la terre d'accueil en évoquant constamment le pays d'origine et les coutumes qui le déterminent. Green affirme que la problématique identitaire est néanmoins sensiblement analogue à celle des écrivaines " pure laine *. De part et d'autre, les femmes veulent trouver leur place et faire entendre leur voix. Les textes des écrivaines québécoises comme ceux des écrivaines d'ailleurs explorent la thématique des relations intergénérationnelles entre les femmes silencieuses du passé et celles ayant la possibilité, aujourd'hui, de s'exprimer : "With pure laine writers like Noël, Théoret, Ouellette-Michalska, and Anne Hébert, they limmigrant writersl share the project of giving voice to silenced women of preceding generations whom they see as essential to the construction of a woman's identity that can only be fully understood in the relationship to the past. " (p. 153-154). Force est de reconnaître que la nouvelle réalité culturelle québécoise est multiethnique, ce qui contribue à la constitution d'une culture postmoderne hétérogène où les marges et la différence font partie du quotidien, comme le propose souvent le discours des minorités dans un contexte postcolonial. Pareil paysage socio-littéraire évoqué dans l'analyse de Green souscrit à la volonté du discours féministe qui cherche à valoriser la pluralité de la subjectivité féminine, posée davantage comme "multiple "que comme "une ", pour reprendre les termes de Diane Lamoureux.

Women \& Narrative Identity reconstitue le parcours des écrivaines québécoises depuis l'avènement des premiers écrits au XIX siècle jusqu'à aujourd'hui. Pour ce faire, Green a revu - révisé - l'écriture des femmes selon une perspective féministe, afin de mettre en lumière les étapes significatives de la cristallisation des récits de l'identité. Si l'auteure insiste sur le fait que la lutte des écrivaines pour s'inscrire dans la tradition littéraire au Québec sera constante, elle présente aussi des analyses qui témoignent d'une bonne compréhension de la quête identitaire menée par les femmes depuis près d'un siècle. Aussi l'accomplissement des écrivaines québécoises consiste-t-il d'abord et avant tout en une libération des schémas narratifs et discursifs imposés par les diktats des hommes. Bien que l'étude de Green expose avec clarté le cheminement des sujets féminins soucieux de laisser leur marque dans le paysage littéraire, elle néglige la contribution au discours identitaire d'auteures 
moins connues. Il aurait été opportun d'effectuer une démarche relevant de la gynocritique afin d'identifier davantage ces voix " mineures " que l'institution littéraire a écartées. En dépit de ces omissions, l'ouvrage parvient à rendre compte de tout un pan de l'évolution de la société québécoise.

Lucie Guillemette et Cynthia Lévesque Université du Québec à Trois-Rivières

\section{Louise Vigneault}

Identité et modernité dans l'art au Québec.

Borduas, Sullivan, Riopelle.

Montréal, Les Cahiers du Québec, 2002.

La question de l'identité au Québec a fait l'objet de nombreuses publications en sciences sociales depuis quelques années déjà. Par contre, rares sont les recherches qui s'interrogent sur la thématique de l'identité québécoise dans l'art. Louise Vigneault tente donc, par cet ouvrage, de combler cette absence en étudiant trois figures de proue de l'art québécois : Paul-Émile Borduas, Françoise Sullivan et Jean-Paul Riopelle.

Dès sa brève introduction, Louise Vigneault présente une approche multidisciplinaire remarquable; l'histoire de l'art se voit enrichie par des emprunts aux principales sciences sociales: l'histoire, l'anthropologie, l'ethnologie, la sociologie, les sciences politiques et la philosophie. C'est en fonction de cette perspective large que l'auteure définit le concept d'identité du sujet moderne principalement dans sa relation à l'Autre. D'abord centrée sur le sujet moderne en général, sa définition de l'identité glisse rapidement vers le contexte québécois. L'identité canadiennefrançaise y est décrite comme une identité collective aliénée par une non-reconnaissance de l'Autre et placée dans une position de méfiance et de survie. Vigneault situe donc les trois artistes dans ce contexte identitaire particulier ; plus précisément, l'auteure relève le dilemme des artistes entre une position moderniste en art - qui tend vers l'autoréférentialité du médium artistique - et l'affirmation d'une identité nationale aux prises avec un mandat de survivance du caractère canadien-français.

Vigneault dresse dans le premier chapitre un portrait socio-politique et historique du milieu artistique depuis le début du siècle dernier jus- 